\title{
Practice Patterns in Orbital Decompression Surgery Among American Society of Ophthalmic Plastic and Reconstructive Surgery Members
}

Sarah W. DeParis · Jing Tian · Fatemeh Rajaii (iD

Received: June 13, 2019 / Published online: August 17, 2019

(c) The Author(s) 2019

\begin{abstract}
Introduction: Orbital decompression surgery for thyroid-associated orbitopathy (TAO) has evolved greatly over the past decades with the development of new surgical techniques and strategies for patient management. However, randomized controlled trials comparing surgical approaches are lacking. The goal of this study was to report the current preferred practices among American Society of Ophthalmic Plastic Surgeons (ASOPRS) members for orbital decompression surgery for TAO, including surgical techniques and perioperative patient management.

Methods: An anonymous electronic survey consisting of 21 questions was sent to the current membership of the ASOPRS, leading to 170 responses in total (response rate: $23.7 \%$ ). Questions regarding preoperative and postoperative care as well as surgical technique were included.
\end{abstract}

Enhanced Digital Features To view enhanced digital features for this article go to https://doi.org/10.6084/ m9.figshare.9073214.

S. W. DeParis · F. Rajaii $(\square)$

Department of Ophthalmology, Wilmer Eye Institute, Johns Hopkins Hospital, Baltimore, MD, USA

e-mail: frajaii1@jhmi.edu

J. Tian

Department of Biostatistics, Johns Hopkins

Hospital, Baltimore, MD, USA
Results: $54.2 \%$ of the respondents prefer twowall surgery as their initial procedure of choice. Of these, $53.8 \%$ prefer balanced lateral and medial decompression as the two-walled procedure of choice, and $44.0 \%$ prefer the orbital floor and medial wall. Steroids are routinely used preoperatively by $35.9 \%$ and postoperatively by $69.2 \%$. Antibiotics are used preoperatively by $32.9 \%$ and postoperatively by $56.4 \%$ of respondents.

Conclusion: Practice patterns for orbital decompression surgery continue to vary widely among ASOPRS members, but balanced medial and lateral wall decompression has gained in popularity as compared to prior studies. Postoperative steroids are preferred by a majority of members.

Keywords: Decompression surgery; Graves' orbitopathy; Orbit; Proptosis; Thyroid eye disease

\section{INTRODUCTION}

Surgical decompression for thyroid-associated orbitopathy (TAO), also known as Graves' orbitopathy, is employed for the correction of disfigurement or exposure keratopathy resulting from proptosis, or for the treatment of dysthyroid optic neuropathy. There has been a great deal of refinement of surgical techniques over the past few decades, but surgical approaches 
vary widely between institutions and individual practitioners.

Decompression of the lateral orbital wall was first described by Dollinger in 1911 [1]. Throughout the following decades, numerous additional techniques were described, including removal of the orbital floor, medial wall, or both the floor and medial wall through a transantral approach [2]. Three-wall decompression was first described by Tessier in 1969 [3]. A 1985 survey of American Society of Ophthalmic Plastic and Reconstructive Surgery (ASOPRS) and Orbital Society members reported on 531 decompression cases performed by 75 surgeons. At that time, $75 \%$ of the cases performed were decompression of the medial wall and orbital floor, and $20 \%$ were three-wall orbital decompression [4].

In 1989, Leone and coworkers described balanced decompression of the medial and lateral orbital walls with removal of the lateral orbital rim as a technique with potentially lower risk for postoperative strabismus [5]. Kennedy first described endoscopic medial wall decompression in 1990 [6]. Further reports of balanced lateral and medial wall removal were later described, both with a transcaruncular approach to the medial orbit or by endoscopic decompression of the medial wall, and without removal of the orbital rim for the lateral wall $[7,8]$. Decompression of the lateral wall only with removal of intraconal fat was also described as an effective procedure for reducing proptosis $[8,9]$.

A survey was conducted in 2014 of common practices in the management of TAO among oculofacial plastic surgeons in the Asia-Pacific region, with a response rate of $25-35 \%$. In this group, $73.5 \%$ of respondents preferred two-wall decompression, with $14.7 \%$ performing onewall and $11.8 \%$ performing three-wall decompression. The wall(s) of choice were not specified [10]. In a 2015 survey of ASOPRS members on the management of severe thyroid eye disease, $38 \%$ of members responded. $61 \%$ of respondents preferred two-wall decompression, with $56 \%$ of these preferring the orbital floor and medial wall and $41 \%$ preferring the medial and lateral orbital walls [11]. A 2016 survey of ASOPRS members with a $17.5 \%$ response rate found that $48.2 \%$ of surgeons preferred a combined orbital floor and medial wall decompression, $39.5 \%$ preferred a balanced lateral and medial decompression, and $11 \%$ preferred single-wall decompression. However, three-wall decompression was not listed as an option in this survey [12].

Complications have been reported to occur at an overall frequency of $9.3 \%$ following orbital decompression surgery, but this may be an underestimation [13]. These complications may include vision loss, hemorrhage, cerebrospinal fluid (CSF) leak, V1 or V2 hypoesthesia, corneal ulcer or other infections, temporal hollowing, or oscillopsia [14]. Various perioperative management techniques have been suggested to prevent such complications, such as preoperative and postoperative steroid and antibiotic treatment, placement of a surgical drain in the orbit, or admission of the patient to the hospital for overnight observation. However, there is a lack of randomized controlled trials studying perioperative management techniques in this setting, and past surveys did not address preoperative or postoperative patient care, so overall practice patterns in this regard are unknown.

Outcomes of orbital decompression were assessed in 2009 by the European Group on Graves' Orbitopathy (EUGOGO), with greater proptosis reduction noted for three-wall decompression as compared to two-wall decompression. Choice of surgical technique was noted to vary widely depending on surgeon preference and experience as well as the culture at a particular institution [15]. Very few randomized controlled trials have studied surgical orbital decompression for TAO. A Cochrane review in 2011 of surgical orbital decompression for thyroid eye disease found two randomized trials for inclusion in the review [16]. The first study compared transantral and endoscopic transantral approaches to decompression and found that the endoscopic transantral approach had similar outcomes but fewer complications, but was limited by a short follow-up period [17]. The second study found that intravenous steroids may be superior to primary surgical decompression for management of compressive optic neuropathy, but this study was limited by 
a small sample size and did not address surgical technique [18]. No randomized controlled trials have compared non-endoscopic surgical techniques or addressed perioperative patient care.

We sought to describe the current practice patterns for orbital decompression surgery among ASOPRS members, including surgical techniques and perioperative patient care.

\section{METHODS}

A survey study was conducted consisting of an anonymous electronic survey with 21 questions. The Johns Hopkins Institutional Review Board reviewed this study and provided it with an IRB exemption. The study adheres to the tenets of the 1964 Declaration of Helsinki and its further amendments. The survey contained a statement of consent that when patients completed the survey, they provided consent to participate. This survey was sent to current ASOPRS members in June 2017. A total of 726 invitations were sent; 10 invitations (1.4\%) were returned, indicating that a total of 716 invitations were received. 170 responses were received, corresponding to a response rate of $23.7 \%$, which is similar to the response rates in previously published surveys on related topics [10-12]. The chi-squared test was performed to test the significance of the correlation between two categorical survey items. A $P$ value of $<0.05$ was considered to indicate statistical significance. The software used for the analysis was SAS, version 9.4 (SAS, Cary, NC, USA).

\section{RESULTS}

Of the 170 respondents, $41.4 \%$ were in private practice without trainees, and $36.1 \%$ were in an academic setting with trainees. $15.4 \%$ were in private practice with trainees, and $7.1 \%$ were in an academic setting without trainees. $57.7 \%$ completed fellowship training more than 15 years ago, 9.4\% completed training 10-15 years ago, $16.5 \%$ completed training 6-10 years ago, and $9.4 \%$ completed training less than 5 years ago. The largest group of respondents perform less than 10 decompression surgeries annually (45.3\%). Only $24.1 \%$ perform more than 20 decompressions per year, and the remainder (30.6\%) perform between 11 and 20 decompressions per year.

Several questions pertained to the preferred wall(s) for a patient's first decompression surgery (Fig. 1). 54.2\% of respondents prefer twowall decompression as their initial surgery of choice. Of the surgeons preferring two-wall decompression, $53.8 \%$ prefer balanced lateral and medial decompression as the two-walled procedure of choice, and $44.0 \%$ prefer the orbital floor and medial wall. $23.2 \%$ of respondents prefer to perform a three-wall decompression of the lateral, inferior, and medial orbital walls as their first surgery. $11.9 \%$ of surgeons perform a single-wall decompression of the lateral wall, and $8.3 \%$ perform a single-wall decompression of the medial wall as the initial procedure of choice. There was no significant relationship between the orbital wall of choice for a patient's first decompression and number of years in practice $(P=0.536)$, practice setting (academic or private practice with or without trainees, $P$ $=0.203$ ), or number of decompression surgeries performed per year $(P=0.398)$.

Additional questions addressed surgical techniques (Fig. 2). 75.9\% of surgeons perform a local anesthetic block. $68.6 \%$ do not use intraoperative navigation for decompression, with $16.5 \%$ of surgeons using this in less than a third of their cases, and $12.4 \%$ using navigation for over two-thirds of their cases. There was no correlation between use of intraoperative navigation and practice setting $(P=0.403)$ or number of years in practice $(P=0.158) .17 .8 \%$ of surgeons routinely remove the lateral orbital rim, with $18.3 \%$ "sometimes" removing the rim. Of those who remove the lateral orbital rim, $76.7 \%$ replace the orbital rim. For decompression of the lateral orbital wall, the most commonly used tool was the high-speed drill, which was preferred by $49.7 \%$ as the only tool used, compared to $13.5 \%$ who used ultrasonic aspiration only. If a conjunctival incision is made, $58.5 \%$ of respondents close the incision. $20 \%$ of surgeons routinely perform a temporary tarsorrhaphy and $3.5 \%$ perform a permanent tarsorrhaphy at the end of surgery. 

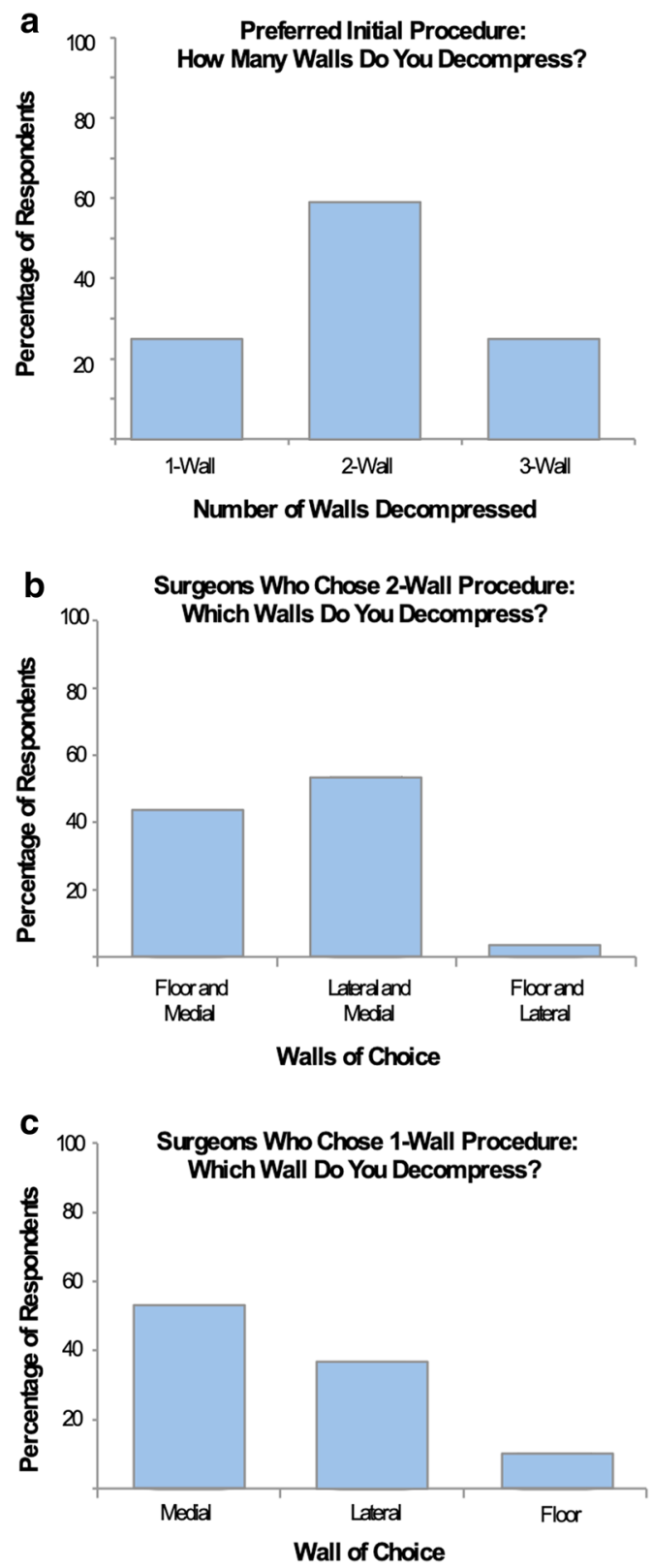

Respondents answered several questions regarding preoperative and postoperative patient care (Fig. 3). Computed tomography (CT) imaging was preferred by the large majority $(95.9 \%)$ of respondents for preoperative imaging evaluation, compared to $3.0 \%$ who prefer magnetic resonance imaging (MRI) and $0.6 \%$ who do not obtain preoperative imaging. Steroids are routinely used preoperatively by $35.9 \%$ of respondents and postoperatively by
4 Fig. 1 Choice of wall for first decompression surgery. Several questions addressed which walls were preferred for a patient's first decompression surgery. a $54.2 \%$ of respondents prefer two-wall decompression as their initial surgery of choice, compared to $23.2 \%$ who perform threewall decompression of the lateral, inferior, and medial orbital walls, and $22.6 \%$ who prefer one-wall decompression. $\mathbf{b}$ Of the surgeons preferring two-wall decompression, $53.8 \%$ prefer balanced lateral and medial decompression, $44.0 \%$ prefer the orbital floor and medial wall, and 2.2\% prefer the floor and lateral wall. $\mathbf{c}$ Of the surgeons who perform one-wall decompression as the initial procedure of choice, $52.6 \%$ decompress the medial wall, $36.8 \%$ decompress the lateral wall, and $10.5 \%$ decompress the floor

$69.2 \%$ of respondents. Antibiotics are used preoperatively by $32.9 \%$ and postoperatively by $56.4 \%$. Only $17.8 \%$ of surgeons routinely place a drain in the orbit at the completion of surgery. $44.4 \%$ of respondents routinely admit patients overnight postoperatively. Regarding timing of the first postoperative examination, $24.3 \%$ of surgeons evaluate the patient on the day of surgery, $37.9 \%$ evaluate the patient on the first postoperative day, and a nearly equivalent portion of $37.3 \%$ see the patient at $2-10$ days after surgery. $73.5 \%$ of respondents do not place an eye patch postoperatively, compared to $22.4 \%$ who place a patch for less than $24 \mathrm{~h}$. Only $4.1 \%$ of surgeons leave an eye patch in place for more than $24 \mathrm{~h}$ after surgery.

\section{DISCUSSION}

Surgical decompression for TAO has evolved greatly since it was first described in 1911. Since then, surgical techniques and preferred practices have changed, and are influenced by surgeon training and institutional tradition [15]. There is a marked lack of randomized controlled trials comparing the outcomes of surgical techniques and perioperative management decisions for orbital decompression surgery [16].

In recent studies, two-wall decompression was generally the most preferred procedure, with the medial orbital wall and the orbital floor being the most common walls of choice $[11,12]$. In this survey, there was a shift to a 

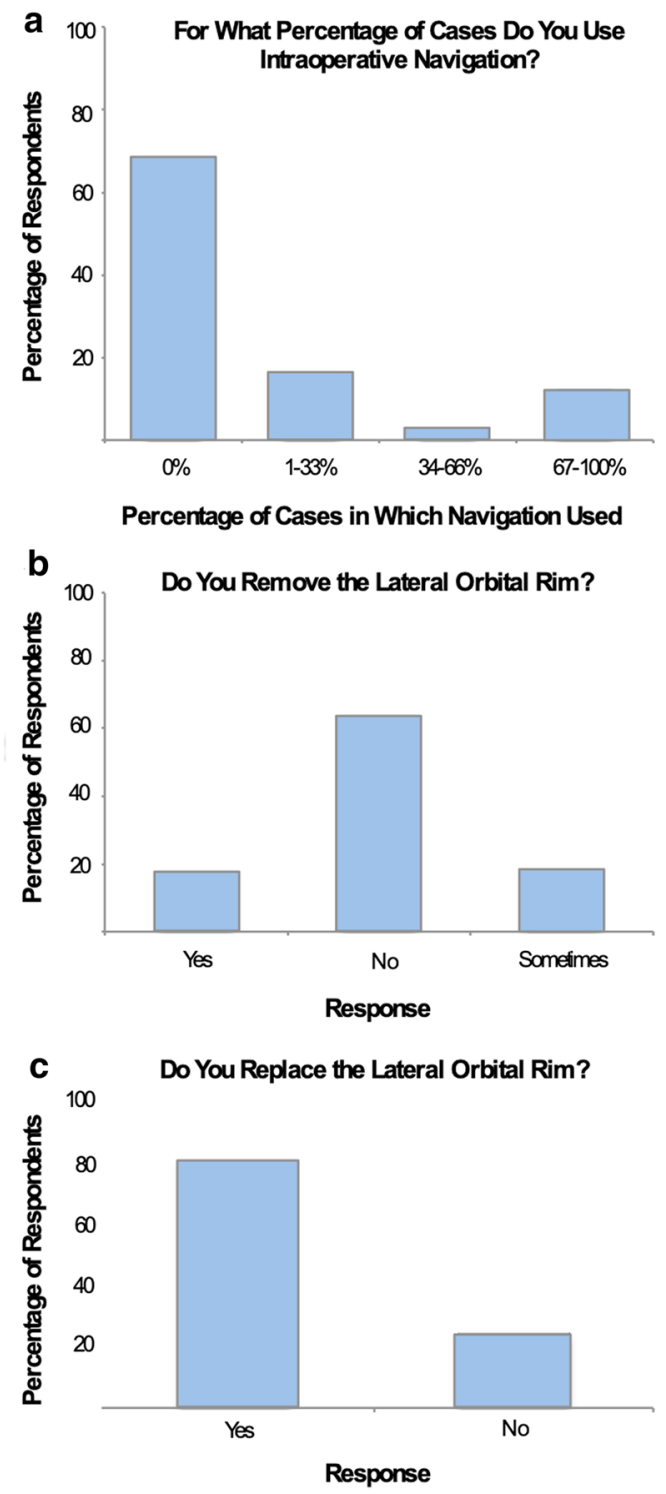

Fig. 2 Surgical decompression technique. Several questions addressed aspects of surgical decompression technique. a $68.6 \%$ of surgeons do not use intraoperative stereotactic navigation. In contrast, $12.4 \%$ of respondents use intraoperative navigation in more than two-thirds of their decompression cases, $2.4 \%$ of respondents use navigation in between one-third and two-thirds of their cases, and $16.6 \%$ use navigation in less than one-third of their cases. b $17.8 \%$ of respondents routinely remove the lateral orbital rim during decompression surgery, $18.3 \%$ remove the lateral rim "sometimes," and $63.9 \%$ of surgeons do not remove the lateral rim. c Of surgeons who do remove the lateral rim, $76.7 \%$ replace the rim following completion of the procedure, compared to $23.3 \%$ who do not larger proportion of surgeons preferring combined lateral and medial decompression as their procedure of choice. There have been a number of studies in recent years describing this socalled "balanced" decompression approach, suggesting that there may be lower rates of postoperative strabismus $[5,7,8,19]$. Such studies may have influenced this trend. However, there was no significant relationship between number of years in practice and preferred wall(s) for initial decompression. Likewise, there was no correlation between the type of practice (academic, private practice, with or without trainee) or number of surgeries performed per year and the preferred wall(s). This may suggest that surgeon preferences are largely influenced by training and institutional tradition.

Stereotactic navigation has been described for orbital decompression surgery as a method allowing the precise removal of the maximum amount of bone during surgery while using a smaller incision, particularly when identifying the safe limits of bony lateral decompression $[20,21]$. However, the use of this technology may be limited by the learning curve involved as well as the high cost of the equipment [20]. Interestingly, there was no correlation between the use of intraoperative navigation and either practice setting or number of years in practice.

The original report of balanced medial and lateral wall decompression by Leone in 1989 described permanent removal of the lateral orbital rim [5]. However, complications such as visible asymmetry, temporal hollowing, or masticatory oscillopsia were reported with this technique [22]. "Rim sparing" decompression was later described; oscillopsia and temporal wasting can occur with this approach as well, though perhaps less frequently [23, 24]. Leaving a thin rim of bone overlying the temporalis muscle has been suggested as a means to prevent these complications [25]. Others described temporary removal of the rim and replacement with either fixation plates or sutures [26, 27]. Decompression of the lateral wall via an intraorbital approach leaving the rim intact may have the advantages of shorter operative time and lower frequency of these complications [28, 29]. In accordance with these 

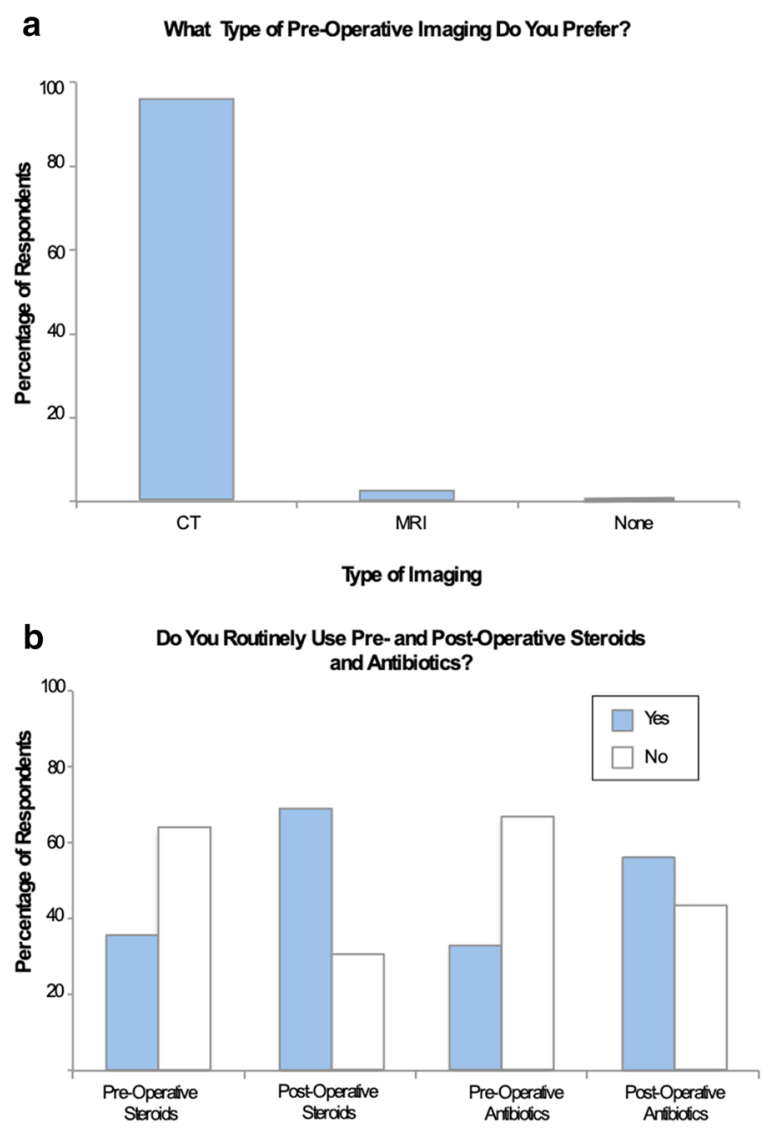

Fig. 3 Preoperative and postoperative management. a The vast majority of respondents obtain preoperative imaging, with $95.9 \%$ preferring CT, and 3\% choosing MRI. Only $0.6 \%$ of surgeons do not obtain preoperative imaging. b Only $35.9 \%$ of respondents routinely use preoperative steroids, compared to $69.2 \%$ who routinely use postoperative steroids. Similarly, only $32.9 \%$ routinely prescribe preoperative antibiotics, and $56.5 \%$ prescribe postoperative antibiotics

findings, the majority of ASOPRS members perform rim-sparing surgery for decompression of the lateral wall $(63.9 \%$ do not remove the rim and $18.3 \%$ remove the rim "sometimes"). Of those who remove the lateral orbital rim, $76.7 \%$ replace it.

When an inferior fornix incision is used to approach the orbit, it has been suggested that foregoing suture closure provides various advantages, including reduced operative time and reduced risk of corneal irritation from sutures or lower eyelid retraction [30]. In this study, $41.5 \%$ of the respondents stated that they do not close a transconjunctival incision if one is made. However, this survey did not differentiate between the transcaruncular approach to the medial wall and the inferior fornix approach, which may influence these results.

Intraorbital hemorrhage can occur with orbital decompression and can lead to vision loss from orbital compartment syndrome. Some have suggested placing a drain in the orbit as a method of prevention, but in this study only $17.8 \%$ of surgeons reported that they routinely do so $[14,21]$. Overnight admission for observation can be considered, and in our survey $44.4 \%$ of surgeons stated that they routinely admit patients following decompression surgery. Placement of an eye patch for 3 days postoperatively has been suggested as a method of further prolapsing orbital tissue into the space created by decompression [21]. Others argue that patch placement does not allow selfmonitoring of postoperative visual acuity, and as such should be avoided. In this study, the majority of surgeons (73.5\%) do not place a patch postoperatively, and if one is placed, the large majority remove the patch less than $24 \mathrm{~h}$ after surgery. Prospective studies are needed to determine how these differences in postoperative management affect patient outcomes.

On review of the literature, little has been studied regarding postoperative and preoperative steroid and antibiotic treatment in the setting of orbital decompression. Intravenous and oral steroids have often been employed for the treatment of active TAO [31]. As many patients undergoing decompression surgery may be in this phase, it makes intuitive sense that prophylactic steroid treatment may reduce the amount of postoperative inflammation. Accordingly, a greater proportion of respondents prescribe postoperative steroids $(69.2 \%)$ than prescribe preoperative steroids (35.9\%). Postoperative infections have been reported following decompression surgery, but the role of prophylactic antibiotics is unknown [14]. We found that ASOPRS members were nearly evenly split in whether they prescribe postoperative antibiotics (56.5\% prescribe), but the majority $(67.1 \%)$ do not prescribe preoperative antibiotics. 


\section{CONCLUSION}

In summary, there is broad variation in both surgical techniques and perioperative patient management among ASOPRS members. This study is limited in that it does not provide information about clinical outcomes of various decompression techniques. Knowledge of current trends among oculofacial plastic surgeons is nevertheless useful in considering surgical approaches. This data will also help to guide studies that compare the outcomes of different decompression methods, which will lead to the further evolution of our practices.

\section{ACKNOWLEDGEMENTS}

Funding. The authors gratefully acknowledge use of the Wilmer Biostatistics Core Grant EY01765. Fatemeh Rajaii is supported by the National Eye Institute of the National Institutes of Health under award number K08EY027093. The Rapid Service Fee was funded by F. Rajaii's discretionary funds.

Authorship. All named authors meet the International Committee of Medical Journal Editors (ICMJE) criteria for authorship for this article, take responsibility for the integrity of the work as a whole, and have given their approval for this version to be published.

Disclosures. Sarah W. DeParis, Jing Tian and Fatemeh Rajaii have nothing to declare.

Compliance with Ethics Guidelines. The Johns Hopkins Institutional Review Board granted approval for this study and designated IRB exemption. The study adheres to the tenets of the Declaration of Helsinki of 1964 and its further amendments. The survey contained a statement of consent that when patients completed the survey, they provided consent to participate.

Data Availability. The datasets generated during and/or analyzed during the current study are available from the corresponding author on reasonable request.

Open Access. This article is distributed under the terms of the Creative Commons Attribution-NonCommercial 4.0 International License (http://creativecommons.org/licenses/ by-nc/4.0/), which permits any noncommercial use, distribution, and reproduction in any medium, provided you give appropriate credit to the original author(s) and the source, provide a link to the Creative Commons license, and indicate if changes were made.

\section{REFERENCES}

1. Dollinger J. Die Druckentlastung der Augenhöhle durch Entfernung der äußeren Orbitalwand bei hochgradigem Exophtalmus (Morbus Basedowii) und konsekutiver Hornhauterkrankung. Dtsch Med Wochenschr. 1911;37:1888.

2. Walsh TE, Ogura JH. Transantral orbital decompression for malignant exophthalmos. Laryngoscope. 1957;67:544-68.

3. Tessier P. Surgical widening of the orbit. Orbits too small. Basedow exophtalmos. Exorbitisms of cranio-facial dysostosis. Congenital anophthalmia, mivrophalmia. Orbital atresia of young enucleasted eyes. Orbital tumors (angioma, meningioma, Recklinghausen). Ann Chir Plast. 1969;14:207-14.

4. McCord CD Jr. Current trends in orbital decompression. Ophthalmology. 1985;92:21-33.

5. Leone CR Jr, Piest KL, Newman RJ. Medial and lateral wall decompression for thyroid ophthalmopathy. Am J Ophthalmol. 1989;108:160-6.

6. Kennedy DW, Goodstein ML, Miller NR, Zinreich SJ. Endoscopic transnasal orbital decompression. Arch Otolaryngol Head Neck Surg. 1990;116:275-82.

7. Kacker A, Kazim M, Murphy M, Trokel S, Close LG. "Balanced" orbital decompression for severe Graves' orbitopathy: technique with treatment algorithm. Otolaryngol Head Neck Surg. 2003;128:228-35.

8. Goldberg RA, Perry JD, Hortaleza V, Tong JT. Strabismus after balanced medial plus lateral wall versus lateral wall only orbital decompression for dysthyroid orbitopathy. Ophthalmic Plast Reconstr Surg. 2000;16:271-7. 
9. McCann JD, Goldberg RA, Anderson RL, Burroughs JR, Ben Simon GJ. Medial wall decompression for optic neuropathy but lateral wall decompression with fat removal for non vision-threatening indications. Am J Ophthalmol. 2006;141:916-7.

10. Sundar G, Chiam N, Lun K, Koh V. Survey of common practices among oculofacial surgeons in the Asia-Pacific region: Graves' orbitopathy. Orbit. 2014;33:319-25.

11. Perumal B, Meyer DR. Treatment of severe thyroid eye disease: a survey of the American Society of Ophthalmic Plastic and Reconstructive Surgery (ASOPRS). Ophthalmic Plast Reconstr Surg. 2015;31:127-31.

12. Reich SS, Null RC, Timoney PJ, Sokol JA. Trends in orbital decompression techniques of surveyed American Society of Ophthalmic Plastic and Reconstructive Surgery members. Ophthalmic Plast Reconstr Surg. 2016;32:434-7.

13. Leong SC, Karkos PD, Macewen CJ, White PS. A systematic review of outcomes following surgical decompression for dysthyroid orbitopathy. Laryngoscope. 2009;119:1106-15.

14. Sellari-Franceschini S, Dallan I, Bajraktari A, et al. Surgical complications in orbital decompression for Graves' orbitopathy. Acta Otorhinolaryngol Ital. 2016;36:265-74.

15. Mourits MP, Bijl H, European Group on Graves' Orbitopathy, et al. Outcome of orbital decompression for disfiguring proptosis in patients with Graves' orbitopathy using various surgical procedures. Br J Ophthalmol. 2009;93:1518-23.

16. Boboridis KG, Bunce C. Surgical orbital decompression for thyroid eye disease. Cochrane Database Syst Rev. 2011;CD007630.

17. Pliego-Maldonado A, Miranda-Ruiz R, VargasAguayo A, Marin-Mendez JA, Pena-Garcia JF, Guerra-Lopez J. Orbit decompression surgery in patients with exophthalmos caused by GravesBasedow disease. Gac Med Mex. 2000;136:11-5.

18. Wakelkamp IM, Baldeschi L, Saeed P, Mourits MP, Prummel MF, Wiersinga WM. Surgical or medical decompression as a first-line treatment of optic neuropathy in Graves' ophthalmopathy? A randomized controlled trial. Clin Endocrinol (Oxf). 2005;63:323-8.

19. Wu CY, Stacey AW, Kahana A. Simultaneous versus staged balanced decompression for thyroid-related compressive optic neuropathy. Ophthalmic Plast Reconstr Surg. 2016;32:462-7.

20. Millar MJ, Maloof AJ. The application of stereotactic navigation surgery to orbital decompression for thyroid-associated orbitopathy. Eye (Lond). 2009;23:1565-71.

21. Mahoney N, Grant MP, Susarla SM, Merbs S. Computer-assisted three-dimensional planning for orbital decompression. Craniomaxillofac Trauma Reconstr. 2015;8:211-7.

22. Fichter N, Krentz H, Guthoff RF. Functional and esthetic outcome after bony lateral wall decompression with orbital rim removal and additional fat resection in Graves' orbitopathy with regard to the configuration of the lateral canthal region. Orbit. 2013;32:239-46.

23. Ueland HO, Haugen $\mathrm{OH}$, Rodahl E. Temporal hollowing and other adverse effects after lateral orbital wall decompression. Acta Ophthalmol. 2016;94:793-7.

24. Siah WF, Patel BC, Malhotra R. Surgical management of temple-related problems following lateral wall rim-sparing orbital decompression for thyroidrelated orbitopathy. $\mathrm{Br} \mathrm{J}$ Ophthalmol. 2016;100:1144-50.

25. Goldberg RA, Kim AJ, Kerivan KM. The lacrimal keyhole, orbital door jamb, and basin of the inferior orbital fissure. Three areas of deep bone in the lateral orbit. Arch Ophthalmol. 1998;116(12):1618-24.

26. Goldberg RA, Hwang MM, Garbutt MV, Shorr N. Orbital decompression for non-Graves' orbitopathy: a consideration of extended indications for decompression. Ophthalmic Plast Reconstr Surg. 1995;11:245-52.

27. Bailey KL, Tower RN, Dailey RA. Customized, single-incision, three-wall orbital decompression. Ophthalmic Plast Reconstr Surg. 2005;21:1-9.

28. Paridaens DA, Verhoeff $K$, Bouwens D, van Den Bosch WA. Transconjunctival orbital decompression in Graves' ophthalmopathy: lateral wall approach $\mathrm{ab}$ interno. $\mathrm{Br} \mathrm{J}$ Ophthalmol. 2000;84:775-81.

29. Kakizaki H, Takahashi Y, Ichinose A, Iwaki M, Selva D, Leibovitch I. The importance of rim removal in deep lateral orbital wall decompression. Clin Ophthalmol. 2011;5:865-9.

30. Lane KA, Bilyk JR, Taub D, Pribitkin EA. "Sutureless" repair of orbital floor and rim fractures. Ophthalmology. 2009;116:135-8.

31. Bartalena L, Baldeschi L, Boboridis $\mathrm{K}$, et al. The 2016 European Thyroid Association/European Group on Graves' Orbitopathy guidelines for the management of Graves' orbitopathy. Eur Thyroid J. 2016;5:9-26. 